\title{
Robust Graph Learning for Misbehavior Detection
}

\author{
Yingtong Dou \\ University of Illinois at Chicago \\ Chicago, Illinois, USA \\ ydou5@uic.edu
}

\begin{abstract}
Recent years have witnessed the thriving of online services like social media, e-commerce, and e-finance. Those services facilitate our daily lives while breeding malicious actors like fraudsters and spammers to promote misinformation, gain monetary rewards, or reap end users' privacy. Graph-based machine learning models have been playing a critical and irreplaceable role in modeling and detecting online misbehaviors. With the observation that misbehaviors are different from massive regular behaviors, the graph models can leverage the relationship between data entities from a holistic view and reveal suspicious behaviors as anomalous nodes/edges/subgraphs on the graph. In this proposal, we investigate the graph-based misbehavior detection models from an adversarial perspective, considering the adversarial nature of malicious actors and real-world factors that impair graph models' robustness. We first introduce two published works enhancing the robustness of several graph-based misbehavior detectors using reinforcement learning. Then, we propose to explore: 1) the robustness of graph neural networks for misinformation detection on social media; and 2) the general robustness of graph neural networks towards unknown perturbations.
\end{abstract}

\section{CCS CONCEPTS}

- Computing methodologies $\rightarrow$ Machine learning; • Information systems $\rightarrow$ Social networks.

\section{KEYWORDS}

Graph Mining; Anomaly Detection; Adversarial Learning

\section{ACM Reference Format:}

Yingtong Dou. 2022. Robust Graph Learning for Misbehavior Detection. In Proceedings of the Fifteenth ACM International Conference on Web Search and Data Mining (WSDM '22), February 21-25, 2022, Tempe, AZ, USA. ACM, New York, NY, USA, 2 pages. https://doi.org/10.1145/3488560.3502213

\section{INTRODUCTION}

The development of online services also cultivates malicious actors who circumvent platforms' regulation to reap money or advocate misinformation $[8,23]$. As an armed race between fraudsters and online platforms, the misbehavior detection algorithms have been

Permission to make digital or hard copies of part or all of this work for personal or classroom use is granted without fee provided that copies are not made or distributed for profit or commercial advantage and that copies bear this notice and the full citation on the first page. Copyrights for third-party components of this work must be honored.

For all other uses, contact the owner/author(s).

WSDM '22, February 21-25, 2022, Tempe, AZ, USA

(C) 2022 Copyright held by the owner/author(s).

ACM ISBN 978-1-4503-9132-0/22/02.

https://doi.org/10.1145/3488560.3502213 developing from tree-based machine learning models to graphbased learning methods. Recently, the Graph Neural Networks (GNNs) have been applied to detecting misbehaviors [20].

Compared to non-graph models, the graph models could leverage the connections between multiple entities interacting with each other and spot the suspicious interactions or groups of entities using graph mining algorithms. For instance, the spammers usually act as a group to post fake reviews to the target item in order to boost the item's rating and reputation on online review platforms like Yelp [18]. If we regard the reviewers and items as two entities in a bipartite graph and the reviews between them as edges, we could observe the grouped spammers above as a densely connected subgraph in contrast to the scattered legitimate reviewer nodes. Many graph mining algorithms have been proposed to discover the suspicious nodes/edges/subgraphs in graphs [7, 16, 18].

With the advent of GNNs [6, 9], recent researchers and practitioners have begun to apply GNNs to misbehavior detection [3, $10,11,22]$. With the assumption that adjacent nodes share similar properties, the GNN model aggregates the information of neighboring nodes recursively to learn the representation of the center node. Given the node representations learned by GNNs, the malicious actor detection problem can be formulated as either a node classification [3] or an anomaly detection problem [22]. Compared to non-deep graph mining algorithms, GNN-based misbehavior detectors can be trained in an end-to-end and semi-supervised fashion with less prior knowledge and feature engineering.

Different from the traditional anomaly detection or node classification problem, which assumes a static distribution of target entities. The malicious actors usually develop their skillset to camouflage themselves and evade the detection of misbehavior detectors. For instance, spammers are adding special characters to reviews as a camouflage [21]. Moreover, real-world evidence shows that intelligent fraud agencies can allocate different assets in a fraud campaign to maximize the campaign outcome with minimum cost [2]. Thus, we must consider the adversarial behavior and the dynamics of the misbehavior detection process. From the model design perspective, a robust misbehavior detector is demanded to combat those intelligent and agile fraudsters. Nonetheless, with the fruitful research on graph-based misbehavior detection, few works have investigated the robustness of misbehavior detectors. In this proposal, we aim to answer the following research questions:

RQ1: How to improve the robustness of non-deep graph-based misbehavior detectors against adversarial fraudsters?

RQ2: How to enhance the GNN-based misbehavior detectors against the camouflaged fraudsters?

RQ3: How robust are the GNN-based misinformation detectors against adversaries?

RQ4: How to handle the concept drift of GNN models due to perturbations induced by fraudsters? 
The first two research questions are answered in our published works [4] and [3], respectively. RQ3 and RQ4 are two proposed research questions. In the remaining sections, I will elucidate the presented works and proposed researches, respectively.

\section{PRESENTED WORK}

RQ1: Robust Spammer Detection via Reinforcement Learning. In this paper [4], considering the real-world spam campaigns ultimately aim at increasing the revenue of dishonest merchants [12], we first propose a practical spamming goal for the spammers, which uses the star rating of the item as a surrogate of its revenue. With the practical spamming goal and different spam detectors using textual or graphical information [18], we propose a multi-agent reinforcement learning framework named Nash-Detect to train a robust spam detector ensemble. Nash-Detect simulates the adversarial game between spammers and defenders. The weights of different spamming strategies and detectors are updated using policy gradient and SGD, respectively.

RQ2: Enhancing GNNs against Camouflaged Fraudsters. Our second research paper [3] is motivated by the camouflaging behavior of real-world fraudsters. In terms of the graph model, a crafty fraudster could build connections to legitimate users to alleviate its suspiciousness. Since the GNNs assume the neighboring nodes share similar properties, the above camouflaging behavior could mislead the GNNs to learn non-discriminative embeddings for camouflaged fraudsters. To enhance GNNs' robustness against camouflaged fraudsters on a multi-relation graph, we first propose a label-aware similarity measure to find the most similar neighbors. Then, we devise a similarity-aware neighbor selector to filter dissimilar neighbors of a center node under a threshold learned by reinforcement learning (RL). Last, we utilize the optimal neighbor filtering thresholds to formulate a relation-aware neighbor aggregator. Furthermore, we extend our model with more RL approaches and show its effectiveness on non-fraud datasets, which have noisy neighbors on the graph [17].

\section{ONGOING AND PROPOSED RESEARCH}

RQ3: Attacking GNN-based Misinformation Detectors. As the online information consumers have confirmation bias and the echo chamber effect [5, 19], using graph to represent news social engagement and identify misinformation through its propagation pattern on social media become a popular approach in misinformation detection. GNNs have been leveraged in misinformation detection in recent years as well $[5,15]$. However, no work has investigated the robustness of GNN-based misinformation detectors while many GNNs have been proved to be vulnerable to adversarial attacks [20,24]. As the fraudsters are actively involved in real-world misinformation campaigns, we propose to devise an adversarial attack framework for GNN-based misinformation detection via simulating the real-world malicious actors. Specifically, we would start from analyzing the news social engagement pattern, then devise dedicated algorithms to probe the vulnerabilities of GNN-based misinformation detectors.

RQ4: Detecting GNN Concept Drift Induced by Fraudsters. The concept drift is a common problem faced by machine learning models on streaming data where the data amount and type continually change. In the misbehavior detection problem, the concept drift can be caused by the tactic shifts of malicious actors or the emergence of new fraudulent types [14]. In other words, the adversarial behavior we studied in [2-4] can result in the concept drift of the static misbehavior detectors. Since most research works only focus on the incremental learning of GNNs $[1,13]$, we propose to characterize concept drift on graphs and devise a general approach to quantify the concept drift induced by unknown perturbations from malicious actors online. We plan to focus on the local neighborhood of each node and vanilla GNNs, and leverage the robustness certification methods or reinforcement learning algorithms [4] to help quantify the influence of concept drift.

\section{ACKNOWLEDGMENTS}

I appreciate the helpful comments made by reviewers toward my proposed research. My research is supported by NSF SaTC-1930941.

\section{REFERENCES}

[1] Y. Cao, H. Peng, J. Wu, Y. Dou, J. Li, and P. S. Yu. 2021. Knowledge-Preserving Incremental Social Event Detection via Heterogeneous GNNs. In $W W W$.

[2] Y. Dou, W. Li, Z. Liu, Z. Dong, J. Luo, and P. S. Yu. 2019. Uncovering Download Fraud Activities in Mobile App Markets.. In ASONAM.

[3] Y. Dou, Z. Liu, L. Sun, Y. Deng, H. Peng, and P. S. Yu. 2020. Enhancing Graph Neural Network-based Fraud Detectors against Camouflaged Fraudsters. In CIKM.

[4] Y. Dou, G. Ma, P. S. Yu, and S. Xie. 2020. Robust Spammer Detection by Nash Reinforcement Learning. In KDD.

[5] Y. Dou, K. Shu, C. Xia, P. S. Yu, and L. Sun. 2021. User Preference-aware Fake News Detection. In SIGIR

[6] W. L. Hamilton and J. Ying, R.and Leskovec. 2017. Inductive representation learning on large graphs. In NeurIPS.

[7] B. Hooi, H. A. Song, A. Beutel, N. Shah, K. Shin, and C. Faloutsos. 2016. FRAUDAR: Bounding Graph Fraud in the Face of Camouflage. In $K D D$.

[8] M. Jiang, P. Cui, and C. Faloutsos. 2016. Suspicious behavior detection: Current trends and future directions. IEEE Intelligent Systems (2016).

[9] T. N. Kipf and M. Welling. 2017. Semi-supervised classification with graph convolutional networks. In ICLR.

[10] Y. Liu, X. Ao, Z. Qin, J. Chi, J. Feng, H. Yang, and Q. He. 2021. Pick and Choose: A GNN-based Imbalanced Learning Approach for Fraud Detection. In $W W W$.

[11] Z. Liu, Y. Dou, P. S. Yu, Y. Deng, and H. Peng. 2020. Alleviating the Inconsistency Problem of Applying Graph Neural Network to Fraud Detection. In SIGIR.

[12] M. Luca and G. Zervas. 2016. Fake it till you make it: Reputation, competition, and Yelp review fraud. Management Science (2016).

[13] Y. Ma, Z. Guo, Z. Ren, J. Tang, and D. Yin. 2020. Streaming graph neural networks. In SIGIR.

[14] T. Mai, K. Hoang, A. Baigutanova, G. Alina, and S. Kim. 2021. Customs Fraud Detection in the Presence of Concept Drift. arXiv preprint arXiv:2109.14155 (2021).

[15] V. Nguyen, K. Sugiyama, P. Nakov, and M. Kan. 2020. FANG: Leveraging Social Context for Fake News Detection Using Graph Representation. In CIKM.

[16] H. Nilforoshan and N. Shah. 2019. SilceNDice: Mining Suspicious Multi-attribute Entity Groups with Multi-view Graphs. In DSAA.

[17] H. Peng, R. Zhang, Y. Dou, R. Yang, J. Zhang, and P. S. Yu. 2021. Reinforced Neighborhood Selection Guided Multi-Relational Graph Neural Networks. TOIS (2021).

[18] S. Rayana and L. Akoglu. 2015. Collective Opinion Spam Detection: Bridging Review Networks and Metadata. In $K D D$.

[19] K. Shu, S. Wang, and H. Liu. 2019. Beyond News Contents: The Role of Social Context for Fake News Detection. In WSDM.

[20] L. Sun, Y. Dou, C. Yang, J. Wang, P. S. Yu, and B. Li. 2018. Adversarial Attack and Defense on Graph Data: A Survey. arXiv preprint arXiv:1812.10528 (2018).

[21] R. Wen, J. Wang, C. Wu, and J. Xiong. 2020. ASA: Adversary Situation Awareness via Heterogeneous Graph Convolutional Networks. In WWW Workshops.

[22] T. Zhao, C. Deng, K. Yu, T. Jiang, D. Wang, and M. Jiang. 2020. Error-Bounded Graph Anomaly Loss for GNNs. In CIKM.

[23] X. Zhou and R. Zafarani. 2020. A survey of fake news: Fundamental theories, detection methods, and opportunities. CSUR (2020).

[24] D. Zügner, A. Akbarnejad, and S. Günnemann. 2018. Adversarial attacks on neural networks for graph data. In $K D D$. 